\title{
An automated sleep-state classification algorithm for quantifying sleep timing and sleep-dependent dynamics of electroencephalographic and cerebral metabolic parameters
}

\author{
This article was published in the following Dove Press journal: \\ Nature and Science of Sleep \\ I September 2015 \\ Number of times this article has been viewed
}

\section{Michael J Rempe ${ }^{1,2}$ \\ William C Clegern ${ }^{2}$ \\ Jonathan PWisor ${ }^{2}$}

'Mathematics and Computer Science, Whitworth University, Spokane, WA, USA; ${ }^{2}$ College of Medical Sciences and Sleep and Performance Research Center, Washington State University, Spokane, WA, USA
Correspondence: Michael J Rempe Mathematics and Computer Science, Whitworth University, $300 \mathrm{~W}$ Hawthorne Road, Spokane, WA, 9925I, USA

Email mrempe@whitworth.edu
Introduction: Rodent sleep research uses electroencephalography (EEG) and electromyography (EMG) to determine the sleep state of an animal at any given time. EEG and EMG signals, typically sampled at $>100 \mathrm{~Hz}$, are segmented arbitrarily into epochs of equal duration (usually 2-10 seconds), and each epoch is scored as wake, slow-wave sleep (SWS), or rapid-eye-movement sleep (REMS), on the basis of visual inspection. Automated state scoring can minimize the burden associated with state and thereby facilitate the use of shorter epoch durations.

Methods: We developed a semiautomated state-scoring procedure that uses a combination of principal component analysis and naïve Bayes classification, with the EEG and EMG as inputs. We validated this algorithm against human-scored sleep-state scoring of data from C57BL/6J and BALB/CJ mice. We then applied a general homeostatic model to characterize the statedependent dynamics of sleep slow-wave activity and cerebral glycolytic flux, measured as lactate concentration.

Results: More than $89 \%$ of epochs scored as wake or SWS by the human were scored as the same state by the machine, whether scoring in 2-second or 10-second epochs. The majority of epochs scored as REMS by the human were also scored as REMS by the machine. However, of epochs scored as REMS by the human, more than $10 \%$ were scored as SWS by the machine and 18 (10-second epochs) to $28 \%$ (2-second epochs) were scored as wake. These biases were not strain-specific, as strain differences in sleep-state timing relative to the light/dark cycle, EEG power spectral profiles, and the homeostatic dynamics of both slow waves and lactate were detected equally effectively with the automated method or the manual scoring method. Error associated with mathematical modeling of temporal dynamics of both EEG slow-wave activity and cerebral lactate either did not differ significantly when state scoring was done with automated versus visual scoring, or was reduced with automated state scoring relative to manual classification.

Conclusions: Machine scoring is as effective as human scoring in detecting experimental effects in rodent sleep studies. Automated scoring is an efficient alternative to visual inspection in studies of strain differences in sleep and the temporal dynamics of sleep-related physiological parameters.

Keywords: EEG, automated scoring, principal component analysis, Bayes classification

\section{Introduction}

In rodent polysomnographic studies, electroencephalographic (EEG) and electromyographic (EMG) data are recorded at a high sampling rate, typically $100 \mathrm{~Hz}$ or more, and then segmented into epochs of arbitrary duration (typically 2-10 seconds in rodents), 
each of which is classified as wake, slow-wave sleep (SWS), or rapid-eye-movement sleep (REMS) on the basis of visual inspection. Although this approach is the gold standard for state scoring, there are several drawbacks: states determined by manual scoring can vary from person to person; when EEG and EMG features fluctuate within a given epoch, the user must assign a single state; and the process is labor intensive and time consuming.

Automated scoring algorithms have been used as an alternative to manual state scoring. Among previously proposed automated scoring approaches are: computing a quantitative state index for each epoch and scoring based on these indices ${ }^{1}$ using principal component analysis (PCA) and manual clustering of epochs in PCA space; ${ }^{2}$ using a naïve Bayes classifier; ${ }^{3}$ using linear discriminant analysis, ${ }^{4}$ using classification trees, ${ }^{4,5}$ using a support vector machine ${ }^{6}{ }^{6}$ using a fully unsupervised Bayesian classifier; ${ }^{7}$ and several methods that use a single channel of EEG data to categorize human sleep. ${ }^{8-10}$ Here we present a hybrid approach to automated scoring of rodent sleep EEG and EMG data in which human-scored epochs are used as a training template for automated state scoring. We combine the PCA approach of Gilmour et $\mathrm{al}^{2}$ with a naïve Bayes classifier that uses the first three principal-component vectors as inputs. Our method is therefore similar to that of Rytkönen et al, ${ }^{3}$ except that we employ the naïve Bayes classifier on the data after constructing manual scoring-derived, state-specific feature vectors and keeping only the first three principal component vectors, rather than splitting up the data into logarithmic frequency bands.

When sleep is scored in epochs, epoch duration is, to an extent, arbitrary. The duration of epochs used often depends on the duration of the sleep-related phenomena of interest in a given study. For instance, in studying brief REMS-related eye movements, 2 -second epochs have been used. ${ }^{11}$ In studying state-switching instability at sleep onset, 4-second epochs have been used when longer epoch durations failed to reveal this instability. ${ }^{12}$ In studying EEG changes across SWSto-REMS transition, a process that evolves gradually over 1-2 minutes, 10-second epochs were used to demonstrate that dynamic. ${ }^{13}$ In studying the gross disruption of sleep/ wake cycles by maternal separation of rodents, 30-second epochs were used. ${ }^{14}$ These examples illustrate that sleep must be studied on different time scales and that machine scoring needs to be validated for these different time scales.

When choosing epoch duration, one balances countervailing factors. The fidelity of state scoring is increased as epoch duration is shortened, as the probability of a state transition occurring within each epoch is thereby reduced. However, the effort associated with state scoring by visual inspection is increased as epoch duration is shortened. Because different research groups use different epoch lengths, it can be difficult to compare sleep data across studies. This issue arises when epoched data has been used in mathematical modeling of sleep-dependent slow-wave dynamics, ${ }^{15-18}$ as the time constants that describe temporal dynamics may be a function of epoch duration.

Here we present a systematic investigation of the effect of epoch length on sleep-dependent dynamics of slow-wave activity (SWA) and lactate concentration in the cerebral cortex. The latter serves as a marker for glucose utilization and varies as a function of sleep state. ${ }^{19-21}$ To investigate the effect of epoch length on these dynamics, we segmented datasets into both 10-second and 2-second epochs. We used the hybrid PCA/ Bayes classifier to automatically score the recordings.

The agreement of a novel automated scoring method with scoring of data by an investigator is a typical performance measure for the novel method. But to the extent that the purpose of scoring sleep is to identify experimental effects of independent variables on dependent variables, whether or not the algorithm detects the same experimental effects as the human scorer is an important consideration as well. Therefore, the algorithm is applied here in two strains of mice and across the entire circadian cycle, in order to determine whether time-of-day effects and differences between the strains in time spent asleep measured by human scoring are also detected algorithmically. Likewise, mathematical modeling with human-scored sleep-state data as an input parameter predicts the dynamics of SWA and lactate concentration over time. ${ }^{22}$ Whether sleep-state data from automated scoring can effectively serve as a predictive parameter for modeling physiological processes is yet another consideration in evaluating a novel autoscoring algorithm.

Our goals for the current study were thus: 1) to determine the agreement between a combined PCA/naïve Bayes autoscoring method and human scoring in measuring sleep timing and sleep homeostatic dynamics; 2) to determine the relative effectiveness of the autoscored and human-scored datasets in detecting experimental effects; and 3) to use this efficient autoscoring approach to determine the effect of 2 -second versus 10-second epoch length on the time dynamics of EEG SWA and lactate concentration.

\section{Methods}

\section{Experimental subjects}

All procedures adhered to National Research Council guidelines (Institute of Laboratory Animal Resources NRC 1996) 
and were approved by the institutional animal care and use committee of Washington State University. Male mice of the $\mathrm{C} 57 \mathrm{BL} / 6 \mathrm{~J}(\mathrm{~B} 6, \mathrm{n}=12)$ and BALB/CJ $(\mathrm{BA}, \mathrm{n}=10)$ strains were used in these experiments. They were purchased at age 8 weeks from Jackson Laboratories (Bar Harbor, ME, USA; strains \#664 and \#651). Animals were housed in a light/dark 12:12 cycle with unrestricted access to standard laboratory chow and water.

\section{Surgical preparation of subjects}

Surgery was performed as described previously, ${ }^{21,23}$ under isoflurane anesthesia. Mice were surgically equipped for bilateral referential frontoparietal EEGs and neck EMGs. Frontal EEG electrode locations were $1.5 \mathrm{~mm}$ lateral to the midline and $1 \mathrm{~mm}$ anterior to bregma. The parietal EEG electrode location was $1.5 \mathrm{~mm}$ left of midline and $2 \mathrm{~mm}$ anterior from lambda. A guide cannula for placement of a lactate biosensor $^{20,21,23}$ was placed in the left frontal cerebral cortex $1.1 \mathrm{~mm}$ anterior and $1.65 \mathrm{~mm}$ lateral from bregma. A dummy stylet was placed in the guide cannula until the day of experimentation, 10-14 days after surgery. Experimentation was completed within 3 weeks of surgical implantation.

\section{Data collection and analysis}

Sleep data were scored by trained experts, as in previous work..$^{21,24,25}$ Epochs in which EMG amplitude was relatively high and EEG amplitude was relatively low were scored as wake. Epochs in which EMG amplitude was relatively low and EEG amplitude relatively high were scored as SWS. Epochs in which both EMG amplitude and EEG amplitude were low and in which theta $(5-9 \mathrm{~Hz})$ activity predominated in the EEG were scored as REMS. Scoring of REMS was additionally context-dependent, in that epochs meeting these criteria that occurred within wake episodes were scored as wake, not REMS.

Lactate was measured using a lactate oxidase based biosensor implanted in the frontal cerebral cortex, as described elsewhere. ${ }^{20,21,23}$ This technique was first published in $1997^{26}$ and has since been refined and commercialized by Pinnacle Technology, Inc. (Lawrence, KS, USA; part \#7004-Lactate). The sensing mechanism consists of a platinum-iridium electrode surrounded by a layer of lactate oxidase molecules. Metabolism of lactate by lactate oxidase produces hydrogen peroxide, which produces a current in the platinumiridium electrode. The current at the sensing electrode is proportionate to the concentration of the substrate for the lactate oxidase enzyme (lactate, the product of glycolysis). Current is monitored at a sampling rate of $1 \mathrm{~Hz}$, providing a moment-by-moment estimate of lactate concentration in the cerebral cortex. Current was averaged across all values within each epoch of data analyzed $(n=10$ for 10 -second epochs; $n=2$ for 2 -second epochs). In order to eliminate noise in the signal from the sensor, each epoch's average value was compared with the mean of the previous ten epoch values. If it deviated from that mean by more than ten standard deviations of that mean, the value for that epoch was replaced by the mean value.

On the day of experimentation, the lactate sensor was precalibrated, as described elsewhere. ${ }^{21,23}$ Approximately 8 hours into the light portion of the light/dark 12:12 cycle, the dummy stylet was removed from the guide cannula and the lactate sensor was inserted into the guide cannula. The uninsulated, enzymatically active portion of the sensor was embedded at a depth of $1 \mathrm{~mm}$ in the cerebral cortex. This depth targets the deeper layers of cerebral cortex, although placement was not verified histologically. The animal was placed into a cylindrical cage, where it remained throughout the duration of the recording session. EEG, EMG, and lactate biosensor current were monitored continuously at $400 \mathrm{~Hz}$ for 40-48 hours thereafter. Data that occurred after insertion of the lactate sensor and before the second SWS episode of 1 minute or longer was not included due to exponential decay of current from the lactate sensor in this equilibrating phase.

For comparison of the effectiveness of human scoring and machine scoring in detecting strain differences in sleep timing across 10-second epochs, 8,640 epochs, representing a complete circadian cycle, were subjected to analysis. Comparing machine scoring of 10 -second epochs to manual scoring in this time range allowed us to determine whether there was a circadian phase-specific bias in the performance of the algorithm. To assess machine-scoring performance relative to human performance in scoring 2-second epochs, we sought to perform analysis on a similar number of epochs as in the 10-second epoch dataset. Each of the epochs used for validation of the algorithm had to be scored by a human, and we anticipated that scoring the numbers of 2-second epochs in one circadian cycle $(43,200$ epochs) would produce significant fatigue in the human scoring the file. This fatigue would decrease the accuracy of the human scoring and thereby confound any measurement of the machine scoring accuracy for 2-second epochs relative to 10 -second epochs. Instead, we reasoned that 8,640 2-second epochs represent 4.8 hours, so we added an additional 360 epochs to make the dataset an even 5 hours. Data from the 5-hour interval between 10 am and 3 pm were used in this analysis because at that time of day rodents transition across all three 
states most reliably, allowing for a thorough assessment of the accuracy of scoring across all three states.

\section{PCA and machine learning}

To autoscore sleep data consisting of EEG and EMG data binned into 2-second or 10-second intervals, we used a combination of PCA and machine learning. The PCA began by constructing seven "feature" vectors from the EEG and EMG data traces, following Gilmour et al. ${ }^{2}$ The seven features are: 1) EEG power in the $1-4 \mathrm{~Hz}$ (delta) range; 2) EEG power in the 5-9 $\mathrm{Hz}$ (theta) range; 3) EEG power in the $10-20 \mathrm{~Hz}$ (low beta) range; 4) EEG power in the 30-40 Hz (high beta) range; 5) EMG power; 6) theta-to-delta ratio; and 7) betato-delta ratio. Each vector contained one element for each epoch in the recording, so each epoch can be thought of as a point in a seven-dimension space. PCA transforms these seven vectors into a set of seven linearly uncorrelated vectors called principal components. The first principal component points in the direction (in seven-dimension space) of greatest variance in the data, and each succeeding component points in the direction of greatest variance with the restriction that it is orthogonal to each of the preceding principal components.

This idea can be visualized in the following manner: because each epoch of data is given a value in seven different categories, it can be visualized as a point in seven-dimension space. If every epoch in a recording is plotted in this seven dimension space, they may form a random cloud of data with no discernible pattern. However, more commonly, when plotted this way the data tend to line up along one or two directions that are not parallel to any of the coordinate axes. The directions along which they line up are the principal components. It is important to note that the principal components are different for each recording, as each one requires a different combination of the seven features to best explain the variation in the data.

Once the principal component vectors were found for the data, we computed the percentage of the total variance in the feature vectors that was explained by each principal component. For every dataset included in this study, the first three principal component vectors accounted for more than $99 \%$ of the variance in the feature vectors, so we reduced the seven-dimension feature space down to three by keeping only the first three principal components. Once this transformation was made, the data were plotted with respect to the first three principal components, and clustering of epochs into wake, non-REM sleep (NREMS), and REMS was clearly visible for each dataset. Moreover, plotting the data using only the first two principal component directions yielded distinct clusters (Figure 1A), indicating that PCA was effectively separating out the sleep states.

We combined this principal component approach with a naïve Bayes classifier to automatically score files. A naïve Bayes classifier requires that a subset of the epochs be scored by a human in order to train the classifier to score the rest of the epochs. The naïve Bayes classifier uses the training data to divide the three-dimensional principal component space into three distinct zones, one for each sleep state. Each unscored epoch is then scored on the basis of which zone it falls into. Finally, as REMS is consistently preceded by non-REMS rather than by wake in wild-type rodents, epochs scored as REMS that were preceded by at least 30 -seconds of wake were rescored as wake. See the Discussion for more details. We implemented both the PCA and the naïve Bayes scoring in MATLAB. Scripts are available to interested parties on request. We first applied the PCA/naïve Bayes hybrid approach to fully-scored 24-hour 10-second epoch data files using the data from 10 am to 2 pm (Zeitgeber time 4-8; a time when all three states alternate on a regular basis) as training for the Bayes scoring algorithm. We used a variety of agreement statistics to compare the scores determined by the algorithm to those made by a human (see Agreement statistics, below). Plotting the machine-scored data along the first two principal component directions yielded images similar to the human-scored data (Figure 1B).

\section{Agreement statistics}

To quantify the agreement of the machine scoring and the human scoring, we calculated five statistical measures. Global agreement is the percentage of total epochs that are scored the same by the human and the algorithm. We also calculated the percentage agreement for wake by counting the number of epochs scored as wake by both human and machine, and divided by the number of epochs scored as wake by the human score. We repeated this calculation for SWS and REMS. Finally, we computed Cohen's unweighted kappa, ${ }^{27}$ a way to quantify agreement while accounting for agreement that would happen due to chance alone.

To determine how many human-scored epochs were needed to successfully train the algorithm to score an entire dataset, we ran the autoscoring procedure using various percentages of the human-scored data as training templates (Figure 1C and D). For each percentage, we randomly selected the appropriate number of human-scored epochs, while ensuring that in each case at least one epoch of each sleep state was present. For both strains, with data binned in 

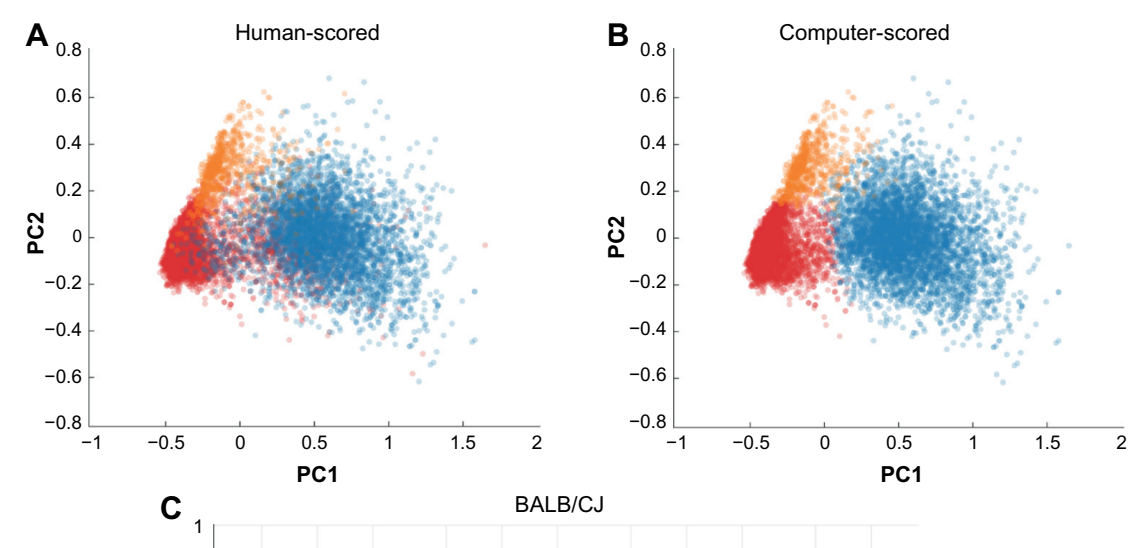

BALB/CJ

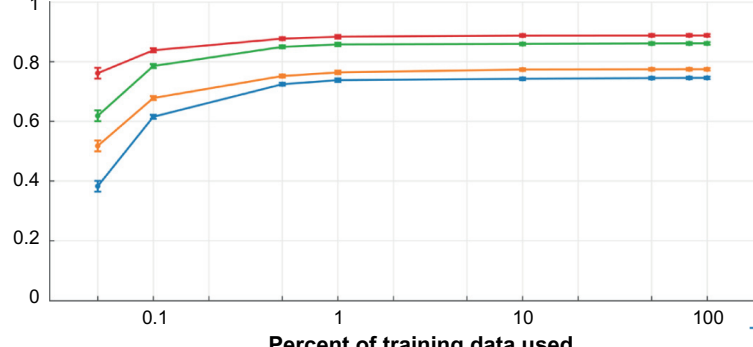

$\rightarrow$ kappa 2 seconds - kappa 10 seconds
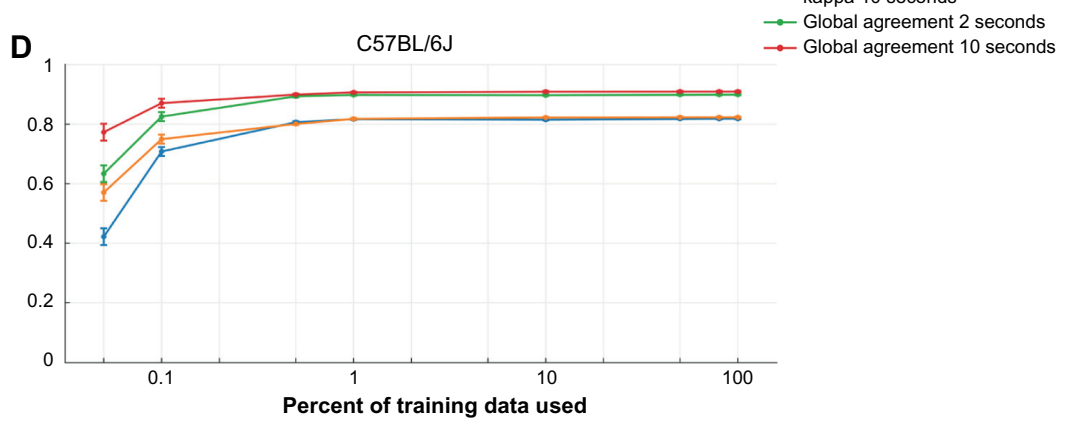

Figure I Comparison of human scoring to machine scoring.

Notes: Principal component plots of a 43-hour recording scored in 10 -second epochs by a human $(\mathbf{A})$ and using the machine learning algorithm (B). Each dot represents one I0-second epoch, and its color represents sleep state (SWS = blue, wake = red, REMS = orange). The computer-scored plot used data from I0 am to 2 pm (Zeitgeber time 4-8 in the first complete light/dark cycle of the recording) as training data to score every epoch in the entire 43-hour recording. Both for BALB/CJ mice (C) and C57BL/6] mice (D), the agreement between machine-scored and human-scored increased as more of the training data were used. For each genetic strain, 8,640 2-second epochs and 8,64010 -second epochs were scored by a human and by the autoscoring procedure, with $0.05 \%, 0.1 \%, 0.5 \%, 1 \%, 10 \%, 50 \%, 80 \%$, and $100 \%$ of those 8,640 epochs used as training data. These correspond to 4 epochs, 9 epochs, 43 epochs, 86 epochs, 864 epochs, 4,320 epochs, 6,912 epochs, and 8,640 epochs, respectively. In each case it was ensured that at least one epoch of each state was present. Two measures of agreement between the human scoring and the machine scoring were computed: Cohen's kappa and global agreement. The x-axis shows the percentage of the 8,640 epochs used as training data, indicating that the learning algorithm requires only about $I \%$ of the training data to reach optimal performance.

Abbreviations: REMS, rapid-eye-movement sleep; SWS, slow-wave sleep.

10 -second epochs the kappa statistic and the global agreement approached $90 \%$, using only about $1 \%$ of the human-scored dataset for training. For each run of the autoscoring algorithm, we used 4 hours of human-scored data as training. This corresponds to $9 \%$ of a 43 -hour recording (as in Figure 1A and B), $16 \%$ of a 24 -hour recording using 10 -second epochs, and $80 \%$ of 8,6402 -second epochs.

\section{Mathematical models of delta activity and lactate dynamics}

We employed a general homeostatic model to quantify the temporal dynamics of both EEG and lactate concentration. ${ }^{22,28}$
To model SWA (the EEG power in the $1-4 \mathrm{~Hz}$ range, called Process S) we assumed that $S$ increases exponentially toward a constant upper asymptote (UA) during wake and REMS, and decreases exponentially toward a constant lower asymptote (LA) during SWS. The time course of S can be found by solving the following differential equation:

$$
\frac{\mathrm{dS}}{\mathrm{dt}}=\sigma\left(\frac{-1}{\tau_{d}}\right)(\mathrm{S}-\mathrm{LA})+(1-\sigma)\left(\frac{-1}{\tau_{i}}\right)(\mathrm{S}-\mathrm{UA})
$$

where $\tau_{i}$ and $\tau_{d}$ are the time constants of the increasing and decreasing exponential functions, respectively, and where 
$\sigma=1$ during an epoch scored as SWS and $\sigma=0$ otherwise. The model for the wake-sleep temporal dynamics of lactate (which we term Process L) is identical to that of Process S, except that UA and LA, respectively, are functions of time rather than constants. The equation is as follows:

$$
\frac{\mathrm{dL}}{\mathrm{dt}}=\sigma\left(\frac{-1}{\tau_{d}}\right)(\mathrm{L}-\mathrm{LA}(\mathrm{t}))+(1-\sigma)\left(\frac{-1}{\tau_{i}}\right)(\mathrm{L}-\mathrm{UA}(\mathrm{t}))
$$

LA and UA were made functions of time to account for the changes in the lactate signal due to the gradual decay in the output of the lactate sensor over the course of the recording. ${ }^{22}$ To compute $\mathrm{UA}(\mathrm{t})$ and $\mathrm{LA}(\mathrm{t})$, we constructed a histogram of the lactate signal in a 2-hour moving window throughout the entire recording. The $90 \%$ level of these histograms was chosen as UA and the 10\% level was chosen as LA. For both the Process S and Process L models the time constants for the rates of increase $\left(\tau_{i}\right)$ and decrease $\left(\tau_{d}\right)$ were optimized, via the Nelder-Mead/simplex method, to minimize the error of the model relative to the raw data. ${ }^{29}$

\section{Results}

\section{Sleep state scoring: human versus machine}

Visual inspection of the machine scoring compared with the human scoring (based on EEG and EMG signals) showed a high degree of agreement (Figure 2). To quantify agreement between the two scoring methods, we computed Cohen's kappa, as well as global agreement and agreement of each of the states (Figure 3). Global agreement and kappa were high for both strains and both epoch lengths. We also pooled the 10-second epoch data and the 2-second epoch data from both strains, and we constructed confusion matrices for each (Table 1) indicating agreement between human scoring and the machine algorithm for each state.
The percentage of correct classifications for each state appears in the entries along the diagonal of the matrix, and misclassifications appear in the off-diagonal entries. For example, the first data row of Table 1 indicates that of all epochs scored as wake by the human scorer, $89.54 \%$ of those were also scored as wake by the algorithm, 9.71\% of those epochs were scored as SWS, and $0.75 \%$ were scored as REMS.

When data were processed in 10-second epochs, the scoring algorithm scored more epochs as SWS than did human scoring (Table $2 ; F_{1,20}=37.6 ; P<0.001$; main effect of scoring method), at the expense of both $\operatorname{REMS}\left(F_{1,20}=54.0 ; P<0.001\right.$; main effect of scoring method) and wake $\left(F_{1,20}=40.0\right.$; $P<0.001$; main effect of scoring method). The absence of a significant time $\times$ scoring method interaction $\left(F_{23,920}=1\right.$, $P>0.05)$ for any of the three states indicates that there was not a time-of-day-specific bias in the disagreement between scoring methods. Of epochs scored as wake by a human, 10\% in BA and 10\% in B6 were not scored as wake by machine. The human versus machine scoring discrepancy resulted in a $5 \%$ (B6 mice) to $7 \%$ (BA mice) reduction in total wake time over 24 hours with machine scoring relative to human scoring (Figure 4A and B; main effect of scoring method, $F_{1,20}=40.0$; $P<0.001)$. Of epochs scored as REMS by a human, $33 \%$ in BA and $25 \%$ in B6 were not scored as REMS by machine. Consequently, the number of epochs scored as REMS over 24 hours was reduced by 13\% (BA mice) to 14\% (B6 mice; Figure 4E and F; main effect of scoring method, $F_{1,20}=37.6$; $P<0.001)$. Epochs scored as either of the two desynchronized states by human scoring were more likely to be scored as SWS by machine. Thus, the number of epochs scored as SWS by machine exceeded that of human scoring by $10 \%$ in BA and $13 \%$ in B6 (Figure 4C and D; main effect of scoring method, $\left.F_{1,20}=54.0 ; P<0.001\right)$. Bias in the machine scoring was not strain-specific, as strain $\times$ scoring method interaction was not significant for any of the three states $\left(F_{1,20}=1.6 ; P>0.2\right)$.

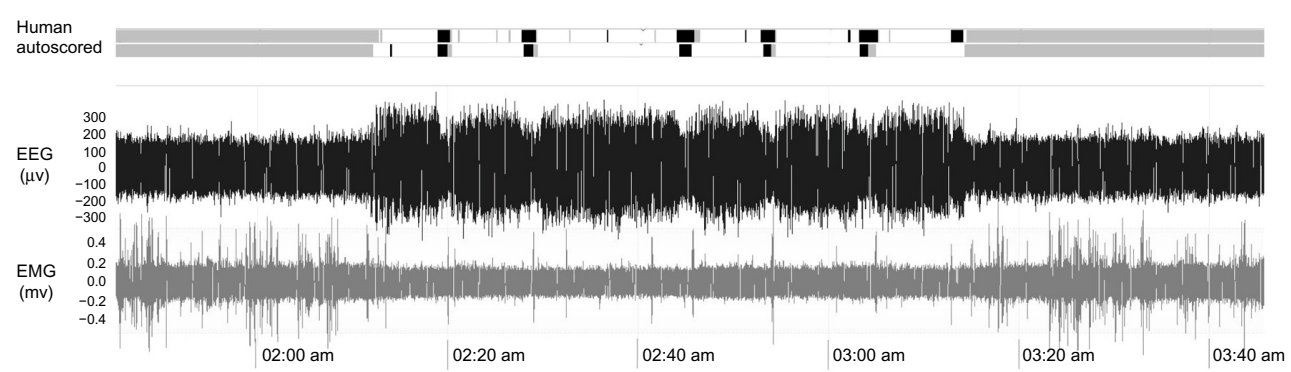

Figure 2 EEG and EMG data for comparison of human scoring and machine scoring.

Notes: The top two traces show human and autoscored sleep states for each I0-second epoch of the EEG and EMG shown in the lower panels. The sleep states are labeled with color (gray for wake, white for SWS, and black for REMS).

Abbreviations: EEG, electroencephalography; EMG, electromyography; REMS, rapid-eye-movement sleep; SWS, slow-wave sleep. 


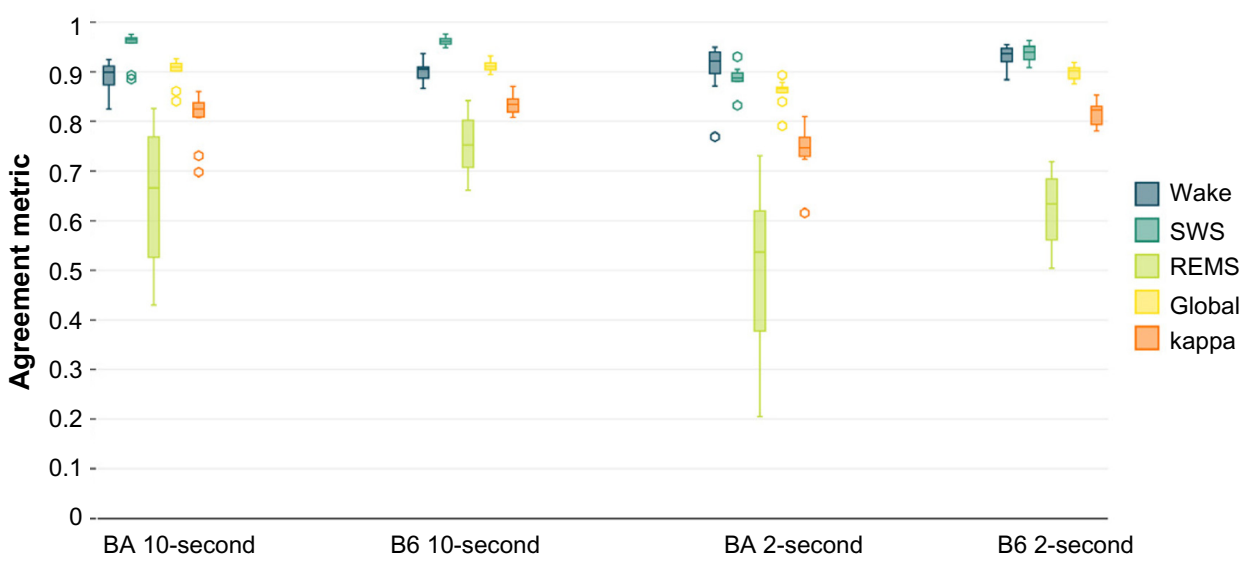

Figure 3 Agreement statistics between human scoring and machine scoring.

Notes: For the data scored in 10-second epochs, the agreement statistics compare the human and machine scoring for the entire 40-48-hour recording. For the data in 2-second epochs, the comparison is between 8,640 epochs that were scored by hand and the same 8,640 epochs scored by the automated scoring algorithm. Lines inside boxes represent median values. The lower end of each box indicates the first quartile of the data (QI), and the upper end represents the third quartile (Q3). To draw the whiskers, we calculated the interquartile range (IQR), which is the distance between QI and Q3. The lower whisker indicates the lowest data point within I.5 IQR of QI. The upper whisker indicates the largest data point within I.5 IQR of Q3. Outliers more than I.5 IQR but less than 3 IQR above Q3 or below QI are represented with open circles. Abbreviations: B6, C57BL/6] mice; BA, BALB/CJ mice; REMS, rapid-eye-movement sleep; SWS, slow-wave sleep.

When data were scored in 2-second epochs, SWS as a percentage of time was no longer significantly affected by scoring method $\left(F_{1,20}=3.3 ; P=0.086\right.$; Figure $5 \mathrm{C}$ and $\left.\mathrm{D}\right)$. However, machine scoring systematically overrepresented wake $\left(F_{1,20}=262.4 ; P<0.001\right.$; Figure $5 \mathrm{~A}$ and $\left.\mathrm{B}\right)$ and underrepresented REMS $\left(F_{1,40}=198.2 ; P<0.001 ;\right.$ Figure 5E and $\left.\mathrm{F}\right)$ relative to human scoring.

\section{EEG power spectra: human versus machine}

EEG power spectra exhibited the typical state dependence irrespective of state-scoring method and epoch duration (Figure 6). In both strains, slow $(<6 \mathrm{~Hz})$ activity predominated in SWS; theta $(6-8 \mathrm{~Hz})$ activity predominated in REMS; EEG amplitude remained relatively low at these frequencies in wake. Although the EEG spectral power curve for machine-scored states largely obscured that of human-scored states in both strains, there were significant

Table I Confusion matrix, human versus machine scoring; percentage of correct classifications

\begin{tabular}{llll}
\hline & $\begin{array}{l}\text { Wake } \\
\text { (machine) }\end{array}$ & $\begin{array}{l}\text { SWS } \\
\text { (machine) }\end{array}$ & $\begin{array}{l}\text { REMS } \\
\text { (machine) }\end{array}$ \\
\hline $\begin{array}{l}\text { Ten-second epochs } \\
\text { Wake (human) }\end{array}$ & 89.54 & 9.71 & 0.75 \\
SWS (human) & 3.31 & 95.58 & 1.11 \\
REMS (human) & 18.66 & 10.89 & 70.45 \\
Two-second epochs & & & \\
Wake (human) & 92.03 & 7.29 & 0.68 \\
SWS (human) & 6.98 & 91.48 & 1.54 \\
REMS (human) & 28.12 & 14.95 & 56.93 \\
\hline
\end{tabular}

Abbreviations: REMS, rapid-eye-movement sleep; SWS, slow-wave sleep. scoring method $\times$ frequency interactions for wake (10-second $F_{19.380}=10.1, P<0.001 ; 2$-second $\left.F_{19.380}=4.0, P<0.001\right)$ and REMS (10-second $F_{19,380}=3.7, P<0.001 ; 2$-second $F_{19,380}=2.2$, $P=0.003$ ), but not SWS. Additionally, $1-4 \mathrm{~Hz}$ SWA was reduced modestly ( $2 \%$ ) but significantly in machine-scored 10-second epochs of SWS relative to human-scored SWS within the B6 strain specifically (Figure 6E, inset).

\section{Measuring sleep state-dependent dynamics of SWA and lactate: human versus machine}

To further investigate the utility of the machine scoring, we employed a homeostatic model to predict the state-dependent dynamics of SWA and cerebral lactate concentration. ${ }^{22} \mathrm{We}$ applied this model to both the human-scored and machinescored data using 10-second epochs and 2-second epochs (Figure 7). The temporal dynamics of the optimized model were unchanged with respect to scoring method using both

Table 2 Sleep states in 10-second epochs, human versus machine scoring (minutes/24 hours)

\begin{tabular}{lllll}
\hline State & Strain & Human & Machine & $\begin{array}{l}\text { ANOVA (scoring } \\
\text { method) }\end{array}$ \\
\hline Wake & BA & $857 \pm 23$ & $800 \pm 20$ & $F_{1,20}=40.0 ; P<0.001$ \\
& B6 & $819 \pm 15$ & $780 \pm 18$ & \\
SWS & BA & $515 \pm 21$ & $582 \pm 20$ & $F_{1,20}=37.6 ; P<0.001$ \\
& B6 & $529 \pm 14$ & $583 \pm 16$ & \\
REMS & BA & $67 \pm 3$ & $58 \pm 4$ & $F_{1,20}=54.0 ; P<0.001$ \\
& B6 & $91 \pm 3$ & $78 \pm 4$ & \\
\hline
\end{tabular}

Abbreviations: ANOVA, analysis of variance; BA, BALB/C] mice; B6, C57BL/6] mice; REMS, rapid-eye-movement sleep; SWS, slow-wave sleep. 

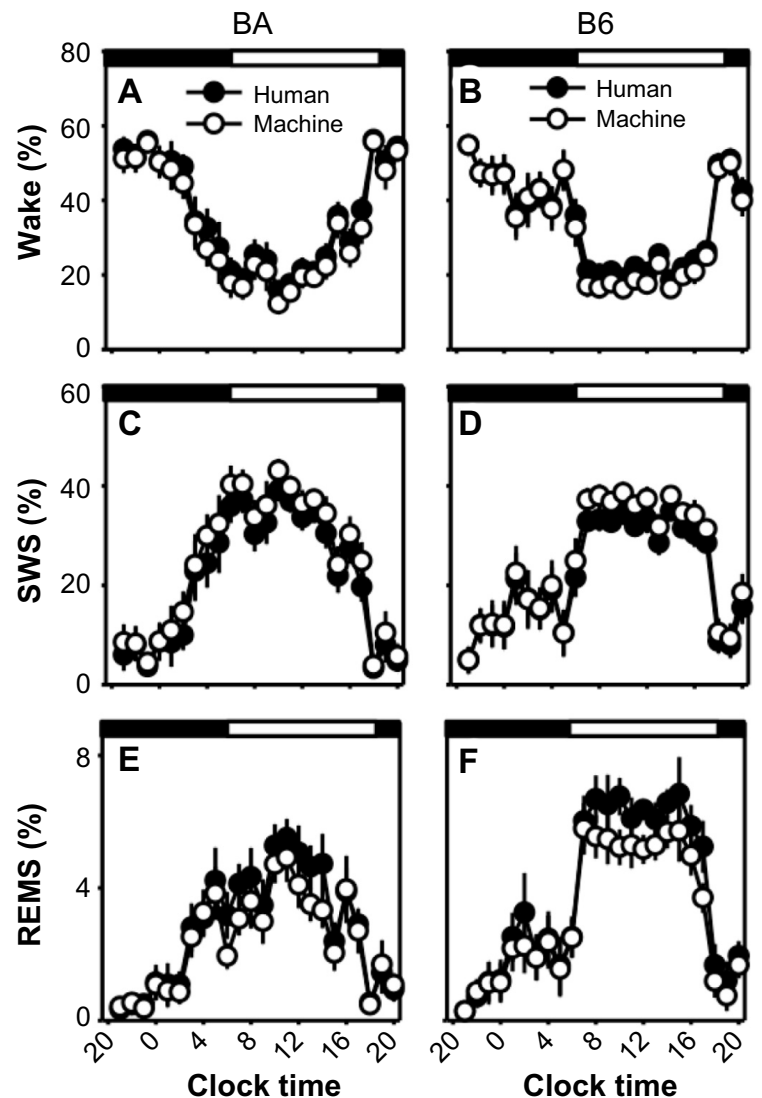

Figure 4 Sleep-state percentages in human-scored versus machine-scored I0-second epoch data.

Notes: Data from the BA strain (left column) and the B6 strain (right column), were binned into 60-minute intervals. Graphs represent the percentage of each interval spent in wake (A and B), SWS (C and D), and REMS (E and F). Open circles represent machine-scored data and filled circles represent human-scored data. Dark and light phases are indicated by black and white bands at the top of each panel. Abbreviations: B6, C57BL/6] mice; BA, BALB/CJ mice; REMS, rapid-eye-movement sleep; SWS, slow-wave sleep.

10-second epochs (Figure 7A, B, E, and F) or 2-second epochs (Figure 7C, D, G, and H). Because only 8,640 of the 2-second epochs were scored by a human, only those data are shown in panels 7A-7D. This corresponds to 4.8 hours of data starting at $10 \mathrm{am}$. The dynamics of the lactate signal were minimally affected by the choice of 2 -second or 10 -second epoch length. In panels 7E-7H, the homeostatic model was fit to delta power $(1-4 \mathrm{~Hz})$ in 5-minute episodes made up of at least $90 \%$ SWS. Although the model of delta power also used 8,640 epochs as training for the machine scoring, all of the data are shown since the temporal dynamics of SWA are slower than those of lactate.

In modeling sleep-state SWA dynamics there was a significant effect of scoring method on both $\tau_{i}$ (the optimized time constant for the rise of SWA as a function of prior time spent in desynchronized states; Figure 8A and C) and $\tau_{d}$ values (the optimized time constant for the decline of SWA as a function of prior time spent in SWS; Figure $8 \mathrm{~B}$ and D)
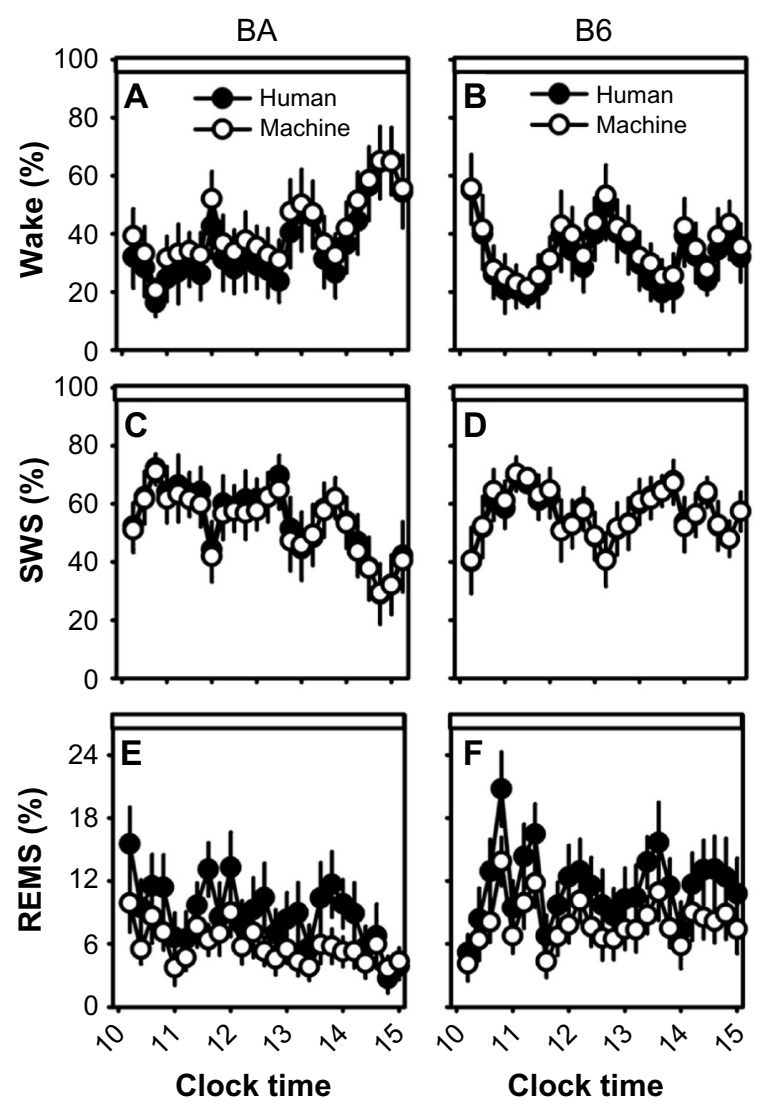

Figure 5 Sleep-state percentages in human-scored versus machine-scored 2-second epoch data.

Notes: Data from the BA strain (left column) and the B6 strain (right column) were binned into 12-minute intervals. Graphs represent the percentage of each interval spent in wake ( $\mathbf{A}$ and $\mathbf{B}$ ), SWS (C and $\mathbf{D})$, and REMS (E and $\mathbf{F}$ ). Open circles represent machine-scored data and filled circles represent human-scored data. Data are from Zeitgeber time 4-9.

Abbreviations: B6, C57BL/6J mice; BA, BALB/CJ mice; REMS, rapid-eye-movement sleep; SWS, slow-wave sleep.

in modeling sleep-state SWA dynamics. Both time constants optimized at higher values in the machine-scored datasets relative to the human-scored datasets, irrespective of strain (strain $\times$ scoring method interaction, $F_{1,20}<0.2, P>0.60$; see strain differences discussed below). In modeling SWA in 10 -second epochs, the mean optimized $\tau_{i}$ values ( 3.2 hours) and $\tau_{d}$ (3.8 hours) across all animals from machine-scored data were greater than those generated from human-scored data ( 2.6 hours, 3.5 hours) by $10 \%$ and $21 \%$, respectively. In modeling SWA in 2-second epochs, the mean optimized $\tau_{i}$ values (4.8 hours) and $\tau_{d}$ (3.8 hours) across all animals from machine-scored data were greater than those generated from human-scored data (4.2 hours, 3.1 hours) by $12 \%$ and $25 \%$, respectively. Scoring method significantly affected $\tau_{d}$ (Figure $8 \mathrm{~F}$ ) but not $\tau_{i}$ values (Figure $8 \mathrm{E}$ ) for the temporal dynamics of lactate concentration in 10 -second epochs. In modeling lactate in 10-second epochs, the mean optimized $\tau_{d}$ values ( 0.23 hours) across all animals from 


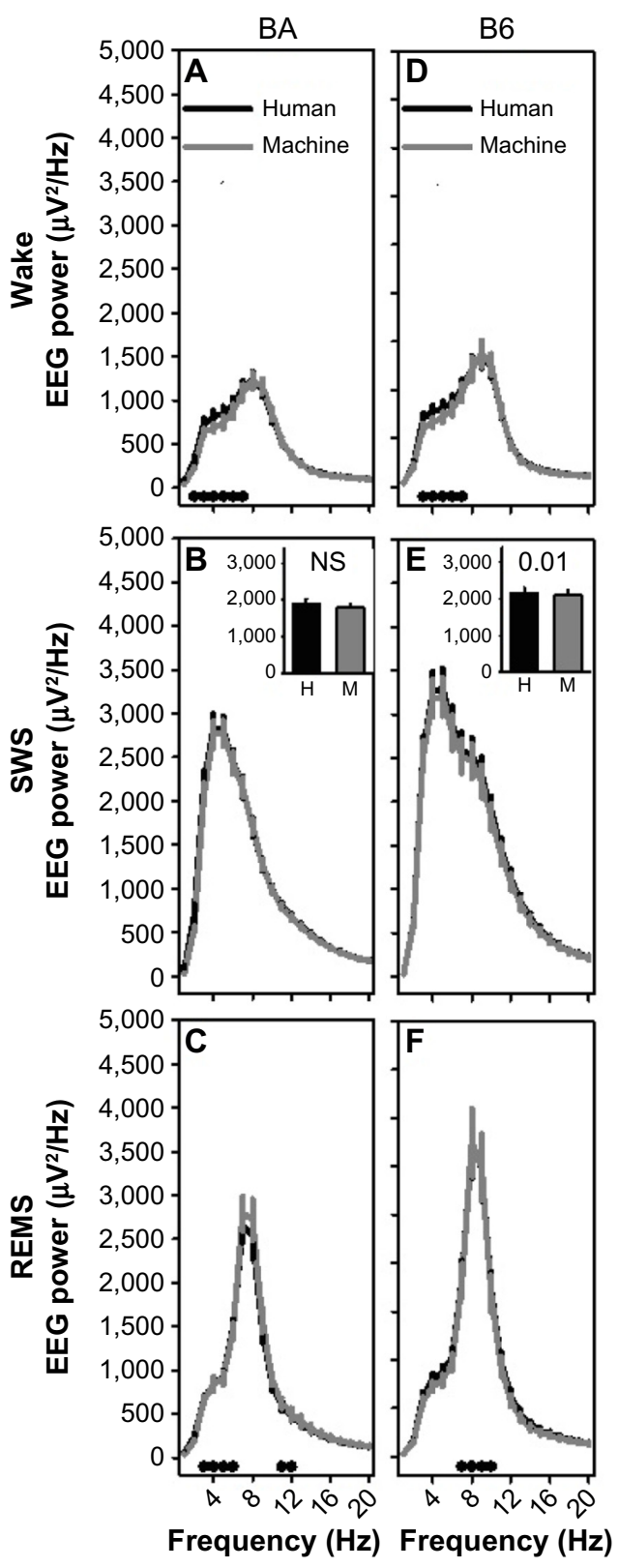

Figure 6 Machine-scored data in 10-second epochs exhibit similar frequency profiles to human-scored data.

Notes: The left column shows data for the BA strain and the right column shows data for the B6 strain. For wake ( $\mathbf{A}$ and $\mathbf{D})$ ), SWS (B and $\mathbf{E}$ ), and REMS ( $\mathbf{C}$ and $\mathbf{F}$ ), the frequency profiles of the human-scored data (black line) and those of the machinescored data (gray line) are shown. The black dots in panels (A, C, D and F) indicate frequency bands in which posthoc comparisons (Fisher's protected least-significant difference) indicated a difference between the curves. The insets in panels ( $\mathbf{B}$ and $\mathbf{E}$ ) show $\mathrm{I}-4 \mathrm{~Hz}$ SWA. $P$-values in these insets indicate main effect of scoring method on SWA.

Abbreviations: $\mathrm{B} 6, \mathrm{C} 57 \mathrm{BL} / 6 \mathrm{~J}$ mice; $\mathrm{BA}, \mathrm{BALB} / \mathrm{CJ}$ mice; $\mathrm{EEG}$, electroencephalography; EMG, electromyography; $H$, human; $M$, machine; NS, not significant; REMS, rapideye-movement sleep; SWA, slow-wave activity; SWS, slow-wave sleep.

machine-scored data were greater than those generated from human-scored data ( 0.18 hours) by $23 \%$. Scoring method did not significantly affect either time constant, $\tau_{d}$ (Figure $8 \mathrm{H}$ ) or $\tau_{i}$ (Figure $8 \mathrm{G}$ ) for the temporal dynamics of lactate concentration in 2-second epochs.
Residuals (Figure 9), a measure of the deviation of optimized mathematical modeling values from the data being modeled, provide an indication of the performance of the model. Lower residuals indicate a better fit to the data being modeled. Two-way analysis of variance with strain as a between-subjects variable and scoring method (automated versus manual) as a within-subjects variable indicated significant effects of scoring method on residual values for modeling lactate dynamics in 10-second epochs (Figure 9C; $\left.F_{1,20}=5.3, P=0.032\right)$ and SWA dynamics in 2-second epochs (Figure 9B; $F_{1,20}=5.3, P=0.032$ ). Scoring method did not significantly affect residuals for lactate dynamics in 2-second epochs (Figure 9D) or SWA dynamics in 10-second epochs (Figure 9A). Residuals did not vary as a function of strain in any of the four analyses, nor were scoring method $\times$ strain interactions significant. Where residuals were affected by scoring method (Figure 9B and C), the residuals were lower (albeit modestly) for automated scoring than for manual scoring. Thus, the performance of automated scoring is as effective as, if not modestly more effective than, manual scoring, for use in modeling sleep state-dependent dynamics of physiological variables.

\section{Machine scoring detects strain differences in sleep timing, EEG power spectra, and sleep-dependent physiological parameter dynamics}

Strain $\times$ scoring method interaction was not significant for any of the three states as a percentage of time, which indicates that there was not a strain-specific bias in the disagreement between scoring methods. The BA and B6 strains, by virtue of genomic differences, exhibit differences in sleep-state timing. Both human and machine scoring detected a significant main effect of strain on REMS as a percentage of time across the entire 24-hour light/dark 12:12 cycle (Table 3 ). Neither wake as a percentage of 24 hours nor SWS as a percentage of 24 hours differed between the two strains regardless of state-scoring method; however, all three states exhibited time-of-day-specific strain effects regardless of whether states were scored by human or machine, as indicated by significant strain $\times$ time interaction for both scoring methods (Table 3).

Posthoc comparisons across strains yielded strikingly similar time-of-day-specific effects of strain on sleep timing in the human-scored and machine-scored datasets (Figure 10). For instance, SWS as a percentage of time differed significantly between strains (Fisher's protected leastsignificant difference) in hours 5, 9, 10, 19, and 24, and at 

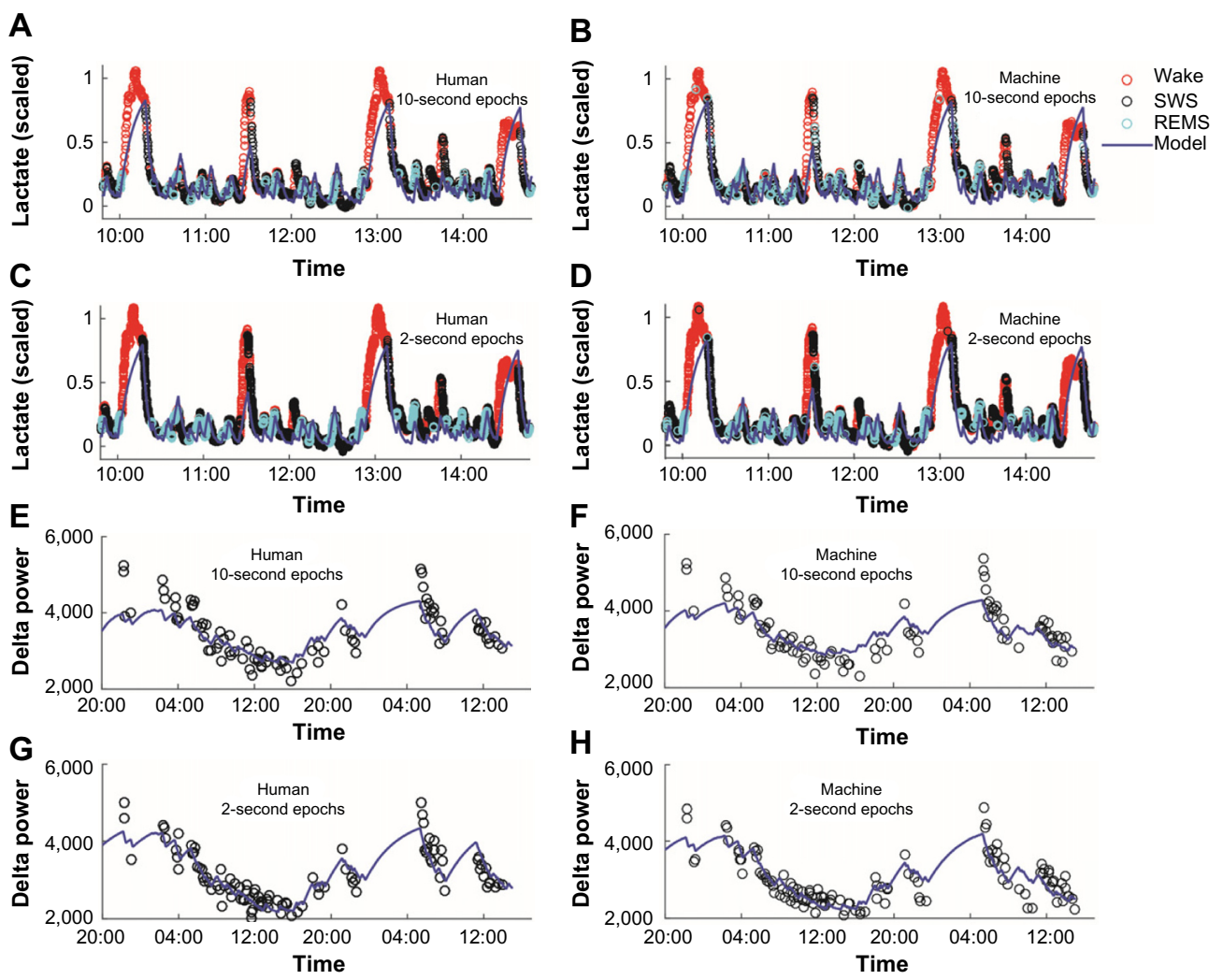

Figure 7 Homeostatic modeling of lactate data and SWA scored by human or machine.

Notes: The left column shows human-scored data and the right-hand column shows machine-scored data. The top four panels show scaled lactate data in I0-second epochs scored by a human (A), 10-second epochs autoscored (B), 2-second epochs scored by a human (C), and 2-second epochs autoscored (D). The bottom four panels show SWA in 5-minute sleep episodes using 10-second epochs scored by a human (E), 10-second epochs autoscored (F), 2-second epochs scored by a human (G), and 2-second epochs autoscored $(\mathbf{H})$. In each panel, a homeostatic model for lactate or SWA was fit to the data (solid curve). The difference in time scale between the lower panels and the upper panels reflects the difference in temporal dynamics of SWA versus those of lactate.

Abbreviations: REMS, rapid-eye-movement sleep; SWA, slow-wave activity; SWS, slow-wave sleep.

no other times, regardless of whether states were scored by human or machine. Wake time differed significantly between strains in hours $5,9,10,19$, and 24 , regardless of whether states were scored by human or machine. REMS time differed significantly between strains in hours 9, 11-13, and 19-21 of recording, regardless of whether states were scored by human or machine. Thus, although machine-based scoring exhibited a significant bias toward SWS, it nonetheless detected the experimental effect of strain on sleep timing. This analysis was not repeated for the 2-second epoch data, as those 8,640 epochs represented a 5-hour window within which daily rhythms of sleep and wake are not apparent.

Repeated-measures analysis of variance indicated significant strain $\times$ frequency interactions affecting spectral profiles of wake, SWS, and REMS in both human-scored and machinescored datasets for both 10-second (Table 4) and 2-second epoch data (not shown). Posthoc comparisons of EEG spectral profiles between strains across $1 \mathrm{~Hz}$ bins indicated that the strain difference detected in the EEG spectral profile in human scored datasets was replicated in the machine-scored datasets (Figure 11). For instance, in 10-second epochs scored as wake, spectral power in the $9-11 \mathrm{~Hz}$ range was elevated in B6 mice relative to BA mice in both the human-scored and machine-scored datasets (Figure 11A and D). In 10-second epochs scored as SWS, spectral power in the 3-12 Hz range was elevated in B6 mice relative to BA mice in both the human-scored and machine-scored datasets (Figure 11B and E). In 10-second epochs scored as REMS, spectral power in the 6-11 Hz range was elevated in B6 mice relative to BA mice in both the human-scored and machine-scored datasets (Figure $11 \mathrm{C}$ and F). These data demonstrate that strain differences in EEG oscillatory activity across sleep states were detected by the machine-scoring method. Similarly, the overlap between scoring methods in detecting strain differences in EEG spectral profiles within the 2-second datasets was near total (data not shown).

The temporal dynamics of both EEG SWA and lactate concentration across 10 -second epochs were strain dependent regardless of scoring method (Figure 8A, B, E, and F). Strain comparison of SWA $\tau_{i}$ and $\tau_{d}$ via Student's $t$-test 

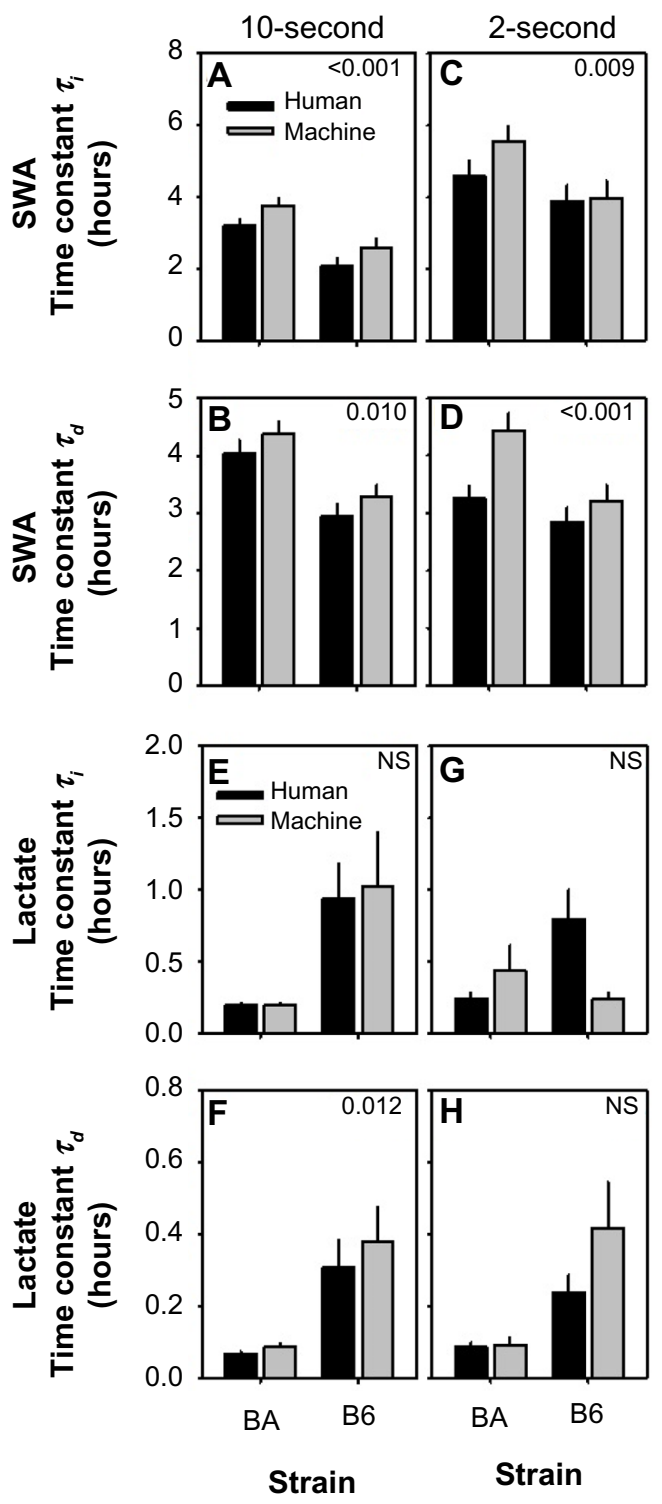

Figure 8 Strain differences in optimal time constants in general homeostatic modeling of state-dependent SWA and lactate dynamics.

Notes: Data represent optimized time constants for wake/REMS-dependent increases $\left(\tau_{i}\right)$ and SWS-dependent decreases $\left(\tau_{\mathrm{f}}\right)$ in SWA $(\mathbf{A}-\mathbf{D})$ and lactate $(\mathbf{E}-\mathbf{H})$ from human-scored data (black bars) and machine-scored data (gray bars). $P$-values are shown for main effect of scoring method on time constants.

Abbreviations: B6, C57BL/6] mice; BA, BALB/CJ mice; NS, not significant; REMS, rapid-eye-movement sleep; SWA, slow-wave activity; SWS, slow-wave sleep.

for independent measures yielded higher values for both parameters in the BA strain relative to the $\mathrm{B} 6$ strain, whether the scoring was automated or manual $\left(\tau_{20}>3.2 ; P<0.004\right.$, Table 5). The strain difference was inverted in the case of lactate-concentration dynamics across 10 -second epochs; time constants for lactate were significantly reduced in BA mice relative to B6 mice (Figure 8E and F). Strain comparison of lactate $\tau_{i}$ and $\tau_{d}$ via Student's $t$-test for independent measures yielded lower values for both parameters in the BA strain relative to the B6 strain, regardless of whether
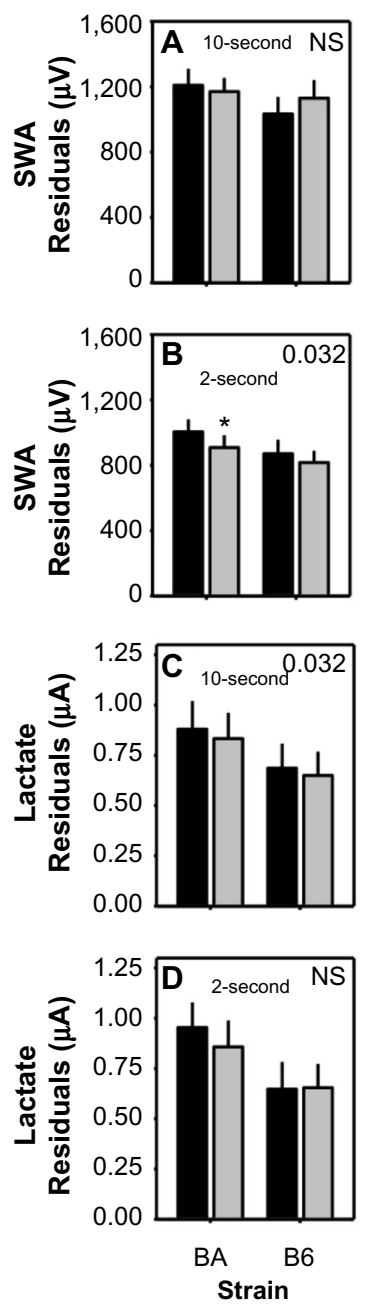

Figure 9 Differences in residuals of model fit to data scored by human or machine. Notes: Data represent the residuals associated with optimized time constants for modeling SWA (A and $\mathbf{B}$ ) and lactate ( $\mathbf{C}$ and $\mathbf{D})$ from human-scored data (black bars) and machine-scored data (gray bars) in either 10-second or 2-second epochs. $P$-values are shown for main effect of scoring method on time constants. Asterisk denotes significant difference for human versus machine scoring within the BA strain (Fisher's protected least-significant difference).

Abbreviations: B6, C57BL/6] mice; BA, BALB/CJ mice; NS, not significant; R SWA, slow-wave activity.

Table 3 Strain difference in sleep timing: human versus machine scoring

\begin{tabular}{lllll}
\hline State & Method & $\begin{array}{l}\text { BA versus } \\
\text { B6, minutes/ } \\
\text { 24 hours }\end{array}$ & $\begin{array}{l}\text { ANOVA } \\
\text { (strain) }\end{array}$ & $\begin{array}{l}\text { ANOVA } \\
\text { (strain } \times \text { time) }\end{array}$ \\
\hline Wake & Human & +38 & $\mathrm{NS}$ & $F_{1,20}=2.5 ; P<0.001$ \\
& Machine & +20 & $\mathrm{NS}$ & $F_{1,20}=2.5 ; P<0.00 \mathrm{I}$ \\
SWS & Human & -14 & $\mathrm{NS}$ & $F_{1,20}=2.6 ; P<0.00 \mathrm{I}$ \\
& Machine & $-\mathrm{I}$ & $\mathrm{NS}$ & $F_{1,20}=2.6 ; P<0.00 \mathrm{I}$ \\
REMS & Human & -24 & $F_{1,20}=27.0 ;$ & $F_{1,20}=2.5 ; P<0.00 \mathrm{I}$ \\
& & & $P<0.00 \mathrm{I}$ & \\
& Machine & -20 & $F_{1,20}=12.0 ;$ & $F_{1,20}=2.5 ; P<0.00 \mathrm{I}$ \\
& & & $P<0.002$ & \\
\hline
\end{tabular}

Abbreviations: ANOVA, analysis of variance; B6, C57BL/6] mice; BA, BALB/C] mice; NS, not significant; REMS, rapid-eye-movement sleep; SWS, slow-wave sleep. 

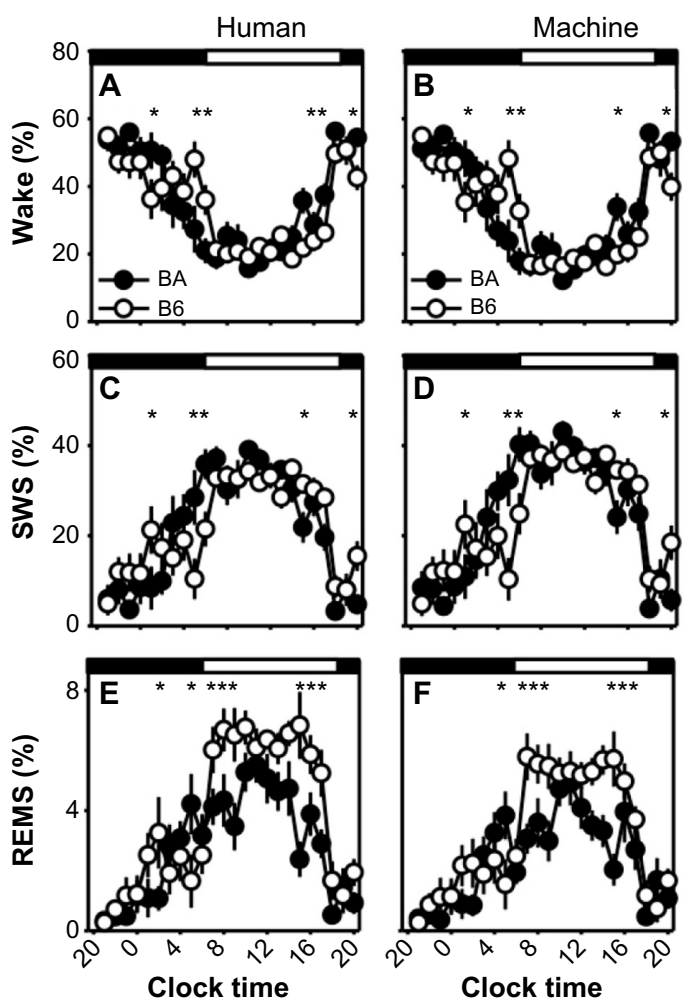

Figure 10 Strain differences in sleep/wake state timing scored in 10-second epochs.

Notes: Strain differences in the time course of sleep/wake states were observed in human-scored data ( $\mathbf{A}, \mathbf{C}$ and $\mathbf{E})$ or machine-scored data (B, D and $\mathbf{F})$. Filled circles represent data from the BA strain, and open circles represent data from the B6 strain. Asterisks indicate 60-minute epochs in which there is a statistically significant difference between strains (Fisher's protected least-significant difference). Timing of the dark and light phases of the 12:12 cycle is indicated by the black and white bars at the top of each graph.

Abbreviations: B6, C57BL/6] mice; BA, BALB/CJ mice; REMS, rapid-eye-movement sleep; SWS, slow-wave sleep.

the scoring was automated or manual $\left(\tau_{20}<-2.1 ; P<0.05\right.$, Table 5). Therefore, even in the presence of a bias toward longer time constants in the machine-scored dataset relative to the-human scored dataset, strain differences in the dynamics of both SWA and lactate were detected across 10-second epochs using both scoring methods.

Table 4 Strain difference in 10 -second EEG spectral profiles: human versus machine

\begin{tabular}{llll}
\hline State & Method & $\begin{array}{l}\text { ANOVA } \\
\text { (strain) }\end{array}$ & $\begin{array}{l}\text { ANOVA } \\
\text { (strain } \times \text { frequency) }\end{array}$ \\
\hline Wake & Human & NS & $F_{19,380}=4.3 ; P=0.00 \mathrm{I}$ \\
SWS & Machine & NS & $F_{19,380}=4.5 ; P=0.00 \mathrm{I}$ \\
& Human & $F_{1,20}=9.0 ; P=0.007$ & $F_{19,380}=7.9 ; P=0.00 \mathrm{I}$ \\
REMS & Machine & $F_{1,20}=9.0 ; P=0.007$ & $F_{19,380}=7.8 ; P=0.00 \mathrm{I}$ \\
& Human & NS & $F_{19,380}=12.8 ; P=0.00 \mathrm{I}$ \\
& Machine & NS & $F_{19,380}=12.8 ; P=0.00 \mathrm{I}$ \\
\hline
\end{tabular}

Abbreviations: ANOVA, analysis of variance; NS, not significant; REMS, rapid-eyemovement sleep; SWS, slow-wave sleep.

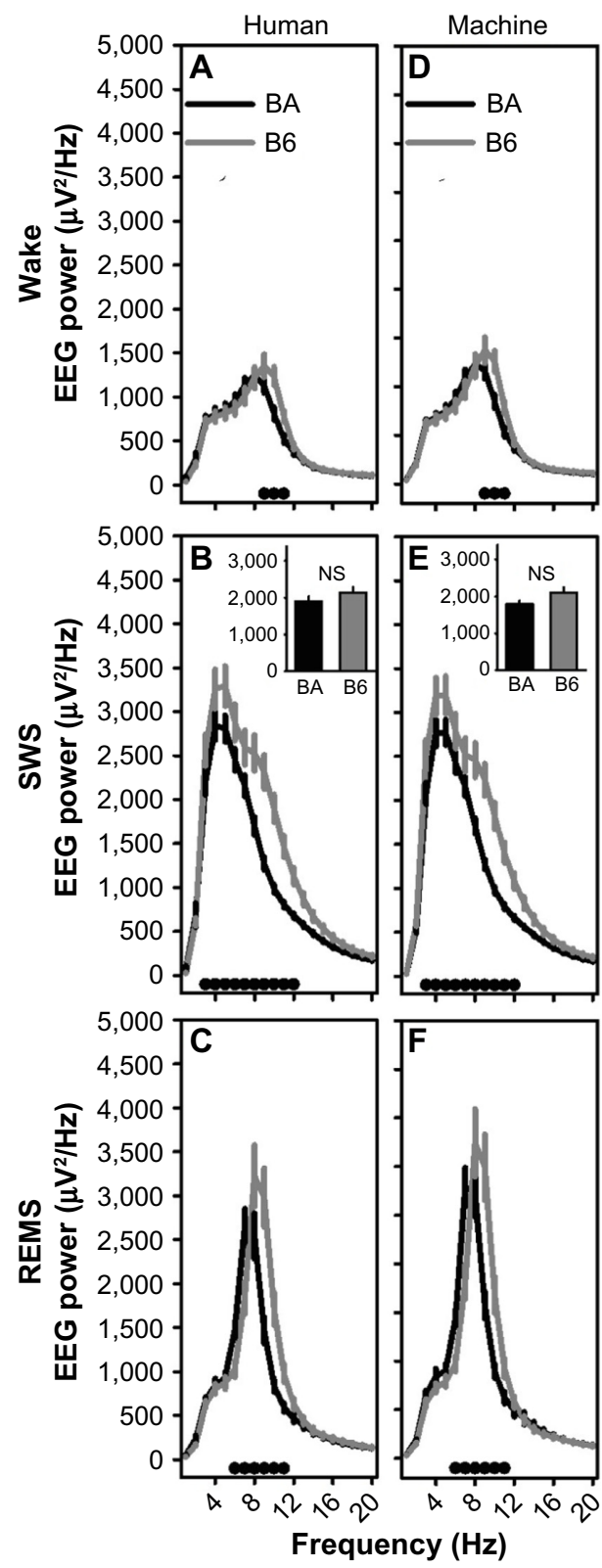

Figure II Strain differences in EEG power spectra in 10-second epochs scored by human or machine.

Notes: The left column shows human-scored data and the right column shows machine-scored data. For wake ( $\mathbf{A}$ and $\mathbf{D})$, SWS (B and E), and REMS (C and F), EEG power differed between strains at specific frequencies. The black dots at the base of each graph indicate frequency bands in which posthoc comparisons (Fisher's protected least-significant difference) indicated a difference between the BA (black line) and B6 (gray line) strains. The insets in panels ( $B$ and (E) show I-4 Hz SWA, which did not differ between strains.

Abbreviations: B6, C57BL/6] mice; BA, BALB/CJmice; EEG, electroencephalography; NS, not significant; REMS, rapid-eye-movement sleep; SWS, slow-wave sleep.

The effect of strain on the temporal dynamics of both EEG SWA and lactate concentration across 2-second epochs was less statistically robust than for 10 -second epochs (Table 5). $\tau_{d}$ for lactate dynamics was the one parameter for which both scoring methods yielded a significant effect of strain (Figure 8H). This parameter was significantly lower in BA 
Table 5 Strain difference in homeostatic dynamics: human versus machine

\begin{tabular}{clll}
\hline Variable & Method & $\tau_{i}$ BA versus B6 & $\tau_{d}$ BA versus B6 \\
\hline $\begin{array}{c}\text { Ten-second } \\
\text { SWA }\end{array}$ & Human & $\tau_{20}=3.7 ; P=0.001$ & $\tau_{20}=3.3 ; P=0.003$ \\
& Machine & $\tau_{20}=3.7 ; P=0.001$ & $\tau_{20}=3.8 ; P=0.001$ \\
Lactate & Human & $\tau_{20}=-3.1 ; P=0.005$ & $\tau_{20}=-3.1 ; P=0.005$ \\
& Machine & $\tau_{20}=-2.2 ; P=0.042$ & $\tau_{20}=-3.0 ; P=0.006$ \\
Two-second & & & \\
SWA & Human & $\tau_{20}=I .2 ; \mathrm{NS}$ & $\tau_{20}=1.3 ; \mathrm{NS}$ \\
& Machine & $\tau_{20}=2.5 ; P=0.020$ & $\tau_{20}=3.0 ; P=0.008$ \\
Lactate & Human & $\tau_{20}=-2.5 ; P=0.019$ & $\tau_{20}=-3.0 ; P=0.007$ \\
& Machine & $\tau_{20}=-1.8 ; \mathrm{NS}$ & $\tau_{20}=-2.5 ; P=0.020$ \\
\hline
\end{tabular}

Abbreviations: B6, C57BL/6] mice; BA, BALB/CJ mice; NS, not significant; SWA, slow-wave activity.

mice than in B6 mice, irrespective of state-scoring method $\left(\tau_{20}<-2.4 ; P<0.05\right.$, Table 5). For the other three parameters assessed, Student's $t$-test values for strain comparison were of equivalent direction (ie, positive for SWA and negative for lactate), if not statistically significant, for both state-scoring methods.

\section{Discussion}

This report introduces a novel method for automated scoring of sleep data. There were discrepancies between humanbased and machine-based scoring, just as there are differences among human raters in state scoring. A more robust assessment of the automated scoring algorithm would compare it against two or more human scorers rather than just one. This would provide a measure of agreement not only between the algorithm and human scorers but also between one human scorer and another. This is a limitation of our approach.

Nonetheless, the utility of the machine-based scoring software introduced here is illustrated by its ability to detect strain differences in sleep-timing parameters, EEG spectral profiles, and sleep-dependent homeostatic variables. The machine-scored dataset detected strain differences in the percent of time spent in each sleep state with hourly resolution; the timing of these strain differences with respect to the light/dark cycle replicated their timing in the human-scored dataset (Figure 10). The strain differences in EEG spectral power that were detected in the human-scored dataset were replicated precisely, at the level of $1 \mathrm{~Hz}$ intervals, in the machine-scored data. Strain differences in time constants associated with sleep-dependent dynamics of SWA and lactate were of equivalent magnitude and direction in the human-scored and machine-scored datasets. Although all of these parameters (sleep-state timing, EEG power spectral profiles, time constants in homeostatic models) varied significantly between the human-scored and machine-scored datasets, scoring method and strain did not exhibit significant interaction in affecting these variables. Thus, the bias in machine-based state scoring was not strain-specific, and strain differences were not lost in the automation process. To the extent that the purpose of state scoring is to detect effects of independent variables on sleep parameters, the automated scoring algorithm introduced here fulfills its purpose in detecting strain differences.

Machine learning alone was not sufficient to accurately score REMS. Some epochs scored as wake by human experts were scored as REMS when automated scoring was based solely on PCA of epochs in isolation. Human raters use contextual cues, specifically the fact that REMS is consistently preceded by a protracted period of SWS in wild-type mice, to discriminate REMS epochs from wake epochs characterized by low EMG and EEG amplitudes occurring against a background of continuous wake. Therefore, we applied contextual information in the algorithmic scoring of REMS: episodes scored as REMS that were preceded by at least 30 seconds of wake were rescored as wake. While not as parsimonious as state scoring based solely on EEG and EMG features of each epoch, this logic replicates the use of contextual information by human experts and in any case increases the level of agreement with human scoring. In experimental circumstances when direct transitions from wake to REMS are expected to occur (such as studies on narcoleptic hypocretin-deficient mice ${ }^{30,31}$ ), the algorithm might be altered to flag the occurrence of such events within the MATLAB interface, allowing for visual inspection and verification of such transitions. However, in the current study of wild-type mice, this consideration is not relevant. Also, while the choice of 30 seconds is reasonable, we acknowledge that it is somewhat arbitrary. In principle, one could determine the optimal amount of preceding wake to use in this rescoring rule by testing several different values and determining the best fit to human-scored data.

We chose to combine the PCA approach with a naïve Bayes classifier because it offers several benefits over other methods. In fact, this was first suggested in a previous study as a possible improvement to current approaches. ${ }^{2}$ First of all, the method is easy to implement in MATLAB using the built-in functions pca.m and classify.m. Second, this approach combines the appealing visualization properties of PCA with the power and efficiency of machine learning. A researcher can visually inspect the effectiveness of the machine scoring by looking at the EEG and EMG traces aligned with the machine score and by looking at the PCA plots of the 
human-scored data and the machine-scored data. Because the method requires scoring a small subset of each data file, this hybrid method is relatively simple, requires only a small subset of each data file to be scored to train the algorithm, and does not overfit the data. We have shown good agreement between human-scored data and machine-scored data when only $10 \%$ of the dataset is scored by hand and used as training for the algorithm. Since the algorithm runs in less than 30 seconds for a 43-hour dataset, the time required to score an entire dataset using this automated approach is about one tenth of the time it would take to score the entire recording by hand.

\section{Implications of 2-second versus 10 -second epoch length and scoring method on time constants for EEG and lactate dynamics}

Using a homeostatic model to quantify EEG SWA dynamics with 10-second epochs scored by a human, we previously found optimal $\tau_{i}$ values that were significantly smaller than those computed by other groups. ${ }^{16}$ To our knowledge, the experimental setup was identical between our recordings and those of Franken et al, ${ }^{16}$ except for the fact that we used an epoch length of 10 seconds and Franken et al used an epoch length of 4 seconds. Because epoch length was the only experimental difference we could find, we speculated that the epoch length may impact the optimal values of the time constants chosen to fit the model to the data. When fitting the model to SWA data (as in Figure 7E-H) following Franken et al, ${ }^{16}$ each data point represents the average delta power in a 5-minute segment that contained at least $90 \%$ SWS. We suspected that the epoch length may affect these data points in the following ways: if a 10-second epoch was part SWS but part wake, the scorer may score it as SWS. Using a finer resolution, a scorer may determine that not all of those 10 seconds should be scored as SWS, which may mean that some 5-minute segments that used to contain at least $90 \%$ SWS were actually less than 90\% and therefore would not create a data point for that 5-minute segment. On the other hand, if some 10-second epochs that were scored as wake actually had some SWS, this may create new data points because there may be 5-minute segments that now have $90 \%$ SWS. Because changing epoch length could add or remove data points, we reasoned that it may alter the time constants of the optimal fit of the model.

The autoscoring algorithm allowed us to quickly rescore 10 -second epoch data into 2 -second epochs, so we could run homeostatic models on the 2 -second data to test this hypothesis. We found that running the model using a shorter epoch length ( 2 seconds) did indeed increase the optimal $\tau_{i}$ values for both strains, making them closer to those found in Franken et al. ${ }^{16}$ However, the optimal value of $\tau_{d}$ did not change for either strain as a function of epoch duration. Application of the automated scoring and modeling techniques to both lactate and SWA in a broader array of strains (like those used by Franken et $\mathrm{al}^{16}$ ) may reveal whether sleep-dependent dynamics of lactate correlate with sleep timing or electroencephalographic parameters.

\section{Conclusion}

We have demonstrated that our machine-scoring algorithm is as effective as human scoring in several different aspects. Various measures of agreement between human scoring and machine scoring were high, and the machine scoring algorithm was just as effective as human scoring in determining subtle differences between genetic strains in both the frequency domain and the time domain. As scoring an entire multiday recording requires less than a minute of computing time (even for 48 hours of 2 -second epoch data), automated scoring is a very efficient alternative to visual inspection in rodent sleep studies and may be similarly effective with sleep data from other species, including humans.

\section{Acknowledgments}

We thank Jonathan P Brennecke for technical assistance. This work was supported by National Institute of Neurological Disorders and Stroke award R01 NS078498 and National Institute of Drug Abuse award R21 DA037708. The authors declare that they have no additional financial support for this work and no off-label or investigational use. All work was performed at Washington State University, Spokane.

\section{Disclosure}

The authors report no conflicts of interest in this work.

\section{References}

1. Stephenson R, Caron AM, Cassel DB, Kostela JC. Automated analysis of sleep-wake state in rats. J Neurosci Methods. 2009;184(2):263-274.

2. Gilmour TP, Fang J, Guan Z, Subramanian T. Manual rat sleep classification in principal component space. Neurosci Lett. 2010;469(1): 97-101.

3. Rytkönen KM, Zitting J, Porkka-Heiskanen T. Automated sleep scoring in rats and mice using the naive Bayes classifier. J Neurosci Methods. 2011;202(1):60-64.

4. Brankack J, Kukushka VI, Vyssotski AL, Draguhn A. EEG gamma frequency and sleep-wake scoring in mice: comparing two types of supervised classifiers. Brain Res. 2010;1322:59-71.

5. Gross BA, Walsh CM, Turakhia AA, Booth V, Mashour GA, Poe GR. Open-source logic-based automated sleep scoring software using electrophysiological recordings in rats. J Neurosci Methods. 2009;184(1): $10-18$. 
6. Crisler S, Morrissey MJ, Anch AM, Barnett DW. Sleep-stage scoring in the rat using a support vector machine. J Neurosci Methods. 2008; 168(2):524-534

7. Libourel PA, Corneyllie A, Luppi PH, Chouvet G, Gervasoni D. Unsupervised online classifier in sleep scoring for sleep deprivation studies. Sleep. 2015;38(5):815-828.

8. Berthomier C, Drouot X, Herman-Stoïca M, et al. Automatic analysis of single-channel sleep EEG: validation in healthy individuals. Sleep. 2007;30(11):1587-1595.

9. Koley B, Dey D. An ensemble system for automatic sleep stage classification using single channel EEG signal. Comput Biol Med. 2012;42(12):1186-1195.

10. Kaplan RF, Wang Y, Loparo KA, Kelly MR, Bootzin RR. Performance evaluation of an automated single-channel sleep-wake detection algorithm. Nat Sci Sleep. 2014;6:113-122.

11. Darchia N, Campbell IG, Feinberg I. Rapid eye movement density is reduced in the normal elderly. Sleep. 2003;26(8):973-977.

12. Moul DE, Germain A, Cashmere JD, Quigley M, Miewald JM, Buysse DJ. Examining initial sleep onset in primary insomnia: a casecontrol study using 4-second epochs. J Clin Sleep Med. 2007;3(5) 479-488.

13. Wisor JP, Wurts SW, Hall FS, et al. Altered rapid eye movement sleep timing in serotonin transporter knockout mice. Neuroreport. 2003;14(2):233-238.

14. Tiba PA, Tufik S, Suchecki D. Effects of maternal separation on baseline sleep and cold stress-induced sleep rebound in adult Wistar rats. Sleep. 2004;27(6):1146-1153.

15. Franken P, Tobler I, Borbély AA. Sleep homeostasis in the rat: simulation of the time course of EEG slow-wave activity. Neurosci Lett. 1991;130(2):141-144.

16. Franken P, Chollet D, Tafti M. The homeostatic regulation of sleep need is under genetic control. J Neurosci. 2001;21(8):2610-2621.

17. Trachsel L, Tobler I, Borbély AA. Electroencephalogram analysis of non-rapid eye movement sleep in rats. Am J Physiol. 1988;255(1 Pt 2): R27-R37.

18. Vyazovskiy VV, Achermann P, Tobler I. Sleep homeostasis in the rat in the light and dark period. Brain Res Bull. 2007;74(1-3):37-44.
19. Dash MB, Tononi G, Cirelli C. Extracellular levels of lactate, but not oxygen, reflect sleep homeostasis in the rat cerebral cortex. Sleep. 2012;35(7):909-919.

20. Naylor E, Aillon DV, Barrett BS, et al. Lactate as a biomarker for sleep. Sleep. 2012;35(9):1209-1222.

21. Wisor JP, Rempe MJ, Schmidt MA, Moore ME, Clegern WC. Sleep slow-wave activity regulates cerebral glycolytic metabolism. Cereb Cortex. 2013;23(8):1978-1987.

22. Rempe MJ, Wisor JP. Cerebral lactate dynamics across sleep/wake cycles. Front Comput Neurosci. 2014;8:174.

23. Clegern WC, Moore ME, Schmidt MA, Wisor J. Simultaneous electroencephalography, real-time measurement of lactate concentration and optogenetic manipulation of neuronal activity in the rodent cerebral cortex. JVis Exp. 2012;(70):e4328.

24. Schmidt MA, Wisor JP. Interleukin 1 receptor contributes to methamphetamine- and sleep deprivation-induced hypersomnolence. Neurosci Lett. 2012;513(2):209-213.

25. Wisor JP, Schmidt MA, Clegern WC. Cerebral microglia mediate sleep/ wake and neuroinflammatory effects of methamphetamine. Brain Behav Immun. 2011;25(4):767-776.

26. Hu Y, Wilson GS. A temporary local energy pool coupled to neuronal activity: fluctuations of extracellular lactate levels in rat brain monitored with rapid-response enzyme-based sensor. J Neurochem. 1997;69(4): 1484-1490.

27. Cohen J. A coefficient of agreement for nominal scales. Educ Psychol Meas. 1960:20(1):37-46.

28. Borbély AA. A two process model of sleep regulation. Hum Neurobiol. 1982;1(3):195-204.

29. Nelder JA, Mead R. A simplex method for function minimization. Comput J. 1965;7(4):308-313.

30. Beuckmann CT, Sinton CM, Williams SC, et al. Expression of a polyglutamine-ataxin-3 transgene in orexin neurons induces narcolepsycataplexy in the rat. $J$ Neurosci. 2004;24(18):4469-4477.

31. Black SW, Morairty SR, Chen TM, et al. GABAB agonism promotes sleep and reduces cataplexy in murine narcolepsy. $J$ Neurosci. 2014;34(19):6485-6494.
Nature and Science of Sleep

\section{Publish your work in this journal}

Nature and Science of Sleep is an international, peer-reviewed, open access journal covering all aspects of sleep science and sleep medicine, including the neurophysiology and functions of sleep, the genetics of sleep, sleep and society, biological rhythms, dreaming, sleep disorders and therapy, and strategies to optimize healthy sleep. The journal welcomes

\section{Dovepress}

original research, clinical \& epidemiological studies, reviews \& evaluations, case reports and extended reports. The manuscript management system is completely online and includes a very quick and fair peerreview system, which is all easy to use. Visit http://www.dovepress.com/ testimonials.php to read real quotes from published authors. 